\title{
The effects of parietal cortex lesions on an object/spatial location paired-associate task in rats
}

\author{
JEFFREY M. LONG \\ National Institute on Aging, National Institutes of Health, Baltimore, Maryland \\ and \\ JERRY E. MELLEM and RAYMOND P. KESNER \\ University of Utah, Salt Lake City, Utah
}

\begin{abstract}
The present experiment was conducted in order to test the hypotheses (1) that the posterior parietal cortex (PPC) serves as a neural system that is critical for binding spatial location and object information in long-term memory and (2) that even restricted lesions of the PPC would result in similar deficits. Long-Evans rats were given either a large or a small PPC lesion or a control surgery under Nembutal anesthesia. After a 1-week recovery period, the rats were tested on either an object or a spatial location go/no-go successive discrimination task. After reaching criterion (a minimum of a 5 sec difference between reward and nonreward trials), they were trained on the other discrimination. After reaching criterion on the second discrimination, all of the rats were trained on a successive discrimination go/no-go task in which they had to remember which object/spatial location pairs had been associated with reward. As compared with controls, neither the small nor the large PPC lesion impaired object or spatial location discrimination. In the paired-associate object/spatial location task, both large and small PPC lesioned rats were impaired, relative to controls. These data suggest that the rodent PPC is not involved in object or spatial location discrimination but rather is involved in discrimination and long-term memory for the combination of object and spatial location information.
\end{abstract}

Behavioral and physiological data have consistently shown that the posterior parietal cortex (PPC) in a variety of species (e.g., humans, nonhuman primates, and rats) is involved in the processing of spatial information. PPC damage in humans (Critchley, 1953; DeRenzi, 1982; Hyvärinen, 1982) or monkeys (Andersen, 1987; Hyvärinen, 1982; Lynch, 1980) can result in a variety of perceptual, motor, or mnemonic impairments involving spatial information. Research on the rodent homologue of the PPC has shown similarities in anatomy (Kolb \& Walkey, 1987; Reep, Chandler, King, \& Corwin, 1994), physiology (Chen, Lin, Barnes, \& McNaughton, 1994; Chen, Lin, Green, Barnes, \& McNaughton, 1994; McNaughton et al., 1994), and behavior (DiMattia \& Kesner, 1988a, 1988b; King \& Corwin, 1992) between the PPCs of both human and nonhuman primates. Previous research on the role of the rodent PPC in spatial processing has mainly focused on the impairment of rats with PPC lesions in solving spatial navigation tasks (e.g., the Morris water maze or the radial-arm maze; DiMattia \& Kesner, 1988a, 1988b; Kolb \& Walkey, 1987). To successfully navigate to a hidden goal in these tasks, it is hypothesized that the rat must possess an internal (central nervous system) representation of the environment (i.e., a spatial cognitive map;

This research was supported by NIH Grant No. 2R01NS20771-13 and NSF Grant No. BNS892-1532. We thank John Thompson and Zhao Gang for running the rats. Correspondence should be addressed to R. P. Kesner, Department of Psychology, University of Utah, Salt Lake City, Utah 84112 (e-mail: rpkesner@behsci.utah.edu).
O'Keefe \& Nadel, 1978; Tolman, 1948). Although the exact nature of this internal representation is a matter of debate, it is thought that the rat must encode, store, and recall information about spatial cues (i.e., distance and spatial location information), landmarks (objects), and the spatial relationships among these cues. Therefore, deficits in spatial navigation tasks after PPC lesions suggest that the PPC is involved in processing spatial cues. However, previous research suggests that the parietal cortex is not critical for either discrimination or memory for single spatial features - for example, allocentric distance (Long \& Kesner, 1996), egocentric distance (Long \& Kesner, in press), or object (landmark) information (Kolb, Buhrmann, McDonald, \& Sutherland, 1994).

In the literature on humans, a recent study suggested that the parietal cortex is involved in binding the color and size of two or more shapes (Friedman-Hill, Robertson, \& Treisman, 1995). Furthermore, a recent PET study in humans suggests that the PPC is involved only when memory for the conjunction of two features is required for each of the individual elements alone. On the basis of these data, one possible role for the rodent parietal cortex could be to bind or to maintain the association between landmark and spatial location information. In other words, the parietal cortex may not be involved in memory for a single landmark or a single spatial location, but rather in the processing that assigns a specific landmark to a specific spatial location. To test this hypothesis, rats with lesions of the parietal cortex were tested in an object/spatial location paired-associate (PA) task that required concur- 
rent memory for both object and spatial location information. In addition, memory for landmark only or spatial location only information was also assessed. A deficit in the PA task (which requires memory for both landmark and spatial location information), in the absence of deficits in either the landmark or the spatial location only memory, would support the idea that the parietal cortex is involved in the memory for the combination of landmark and spatial location information.

Furthermore, two different anatomical locations for the PPC were assessed. This was based on the recent work of Reep and colleagues (Reep et al., 1994), who have suggested a different anatomical definition for the rodent PPC, as compared with that used previously (Swanson, 1992).

\section{METHOD}

\section{Subjects}

Seventeen male Long-Evans rats, approximately 4 months of age at the start of the experiment, served as subjects. The rats were housed individually in metal hanging cages located in a colony with a 12:12-h light:dark cycle. All rats had free access to water, with food restricted to maintain each rat at approximateiy $85 \%-90 \%$ of its free-feeding weight. All testing was conducted during the light portion of the light:dark cycle.

\section{Apparatus}

The apparatus was a Plexiglas box designed to reduce the rat's exposure to extra-maze cues. The four sidewalls of the test box and the internal guillotine doors were constructed of red Plexiglas, designed to block the rat's vision. The box was $30.5 \mathrm{~cm}$ wide, $30.5 \mathrm{~cm}$ deep, and $86 \mathrm{~cm}$ in length. There was a removable guillotine door, which blocked the rat's access to and view of stimuli placed behind the door. The floor consisted of two parts: a permanent base and a 1.5 -cm-thick insert, which was removable in order to aid in cleaning. A $4 \times 5$ matrix of food wells, $2 \mathrm{~cm}$ in diameter, and $1.5 \mathrm{~cm}$ deep, was located at both end sections of the box.

The stimuli were two three-dimensional toy objects that varied on such dimensions as size, shape, color, and texture. Latencies were recorded with a stopwatch, and the food reward was Froot Loops breakfast cereal (Kellogg's).

\section{Procedure}

\section{Presurgical Training}

All of the rats completed pretraining within a 2-week period, with the total time spent at each stage tailored to the individual rat. First the rats were introduced to the testing apparatus, with the food reward placed randomly throughout. The rats were then shaped only to search for food in food wells with an object nearby (not an object used during testing), and, finally, the rats were shaped to displace an object in order to obtain food located in the well underneath. The rats were then randomly assigned to the large PPC lesion group $(n=5)$, the small PPC lesion group $(n=5)$, or the control group $(n=7)$.

\footnotetext{
Surgery

All of the rats were anesthetized with Nembutal $(50 \mathrm{mg} / \mathrm{kg}$, intraperitoneal [i.p.] sodium pentobarbital) and given atropine sulfate $(0.2 \mathrm{mg} / \mathrm{kg}$, i.p. $)$ as a precautionary measure against respiratory distress. Rats were then placed in a Kopf stereotaxic apparatus, an incision was made along the midline, and the skull was exposed.
}

Rats in the large PPC lesion group ( $n=5)$ had the bone removed above the lesion site with bone rongeurs and the dura removed. Cortex was aspirated at coordinates intended to destroy all of the PPC, as labeled in the Swanson atlas (Swanson, 1992). This included the region from $2.0 \mathrm{~mm}$ posterior to bregma to $6.0 \mathrm{~mm}$ posterior to bregma and from $2 \mathrm{~mm}$ lateral of midline to approximately $1 \mathrm{~mm}$ above the rhinal sulcus in the medial-lateral plane. The rats in the small PPC lesion group $(n=5)$ had the cortex aspirated at coordinates intended to destroy the PPC, as defined by Reep et al. (1994). This included the region from $3.5 \mathrm{~mm}$ posterior to bregma to $5.0 \mathrm{~mm}$ posterior to bregma and from $1.5 \mathrm{~mm}$ lateral of midline to approximately $4 \mathrm{~mm}$ lateral of midline. The rats in the control group $(n=7)$ had bone removed on the basis of the coordinates used for the large lesion group.

At the completion of surgery, all of the rats were sutured and placed on a heating pad for a recovery period of approximately $3 \mathrm{~h}$ and then returned to their home cage. During their 7-day recovery period, they were given an antibiotic (tetracycline $20 \mathrm{~g} / \mathrm{liter}$; Venco, Inc., St. Joseph, MO) in their drinking water as a precautionary measure against postoperative infection.

\section{Postsurgery Training}

General. All of the rats were trained on three separate discriminations. The first discrimination was either an object or a spatial location discrimination. The order was randomized for each rat. After reaching criterion level performance on the first discrimination, each rat was then tested on the other discrimination. After reaching criterion level on the second discrimination, each rat was then tested on an object plus spatial location discrimination, until criterion level performance was reached or for 580 trials.

Object discrimination. This experiment required the rats to discriminate between the identities of two toy objects presented at one of two spatial locations. In this task, object information is important, and spatial location information is irrelevant. The two locations were defined by two of the food wells in the $5 \times 4$ matrix (five wells across, four wells deep). The wells that were used were on either end of the row that was second from the back of the apparatus. The actual separation of the spatial locations was $3 \mathrm{~cm}$, with both locations being an equal distance from the start door. Two different toy objects served as stimuli. One object was always paired with food, whereas the other was never paired with food, regardless of which of the two spatial locations the object occupied. The specific reward valence for any one stimulus was counterbalanced across rats. This was a go/no-go successive discrimination task. The rat was located at one end of the apparatus, with its view of and access to the stimuli blocked by the door. On each trial, the rat was presented with one of the two objects, counterbalanced between the two spatial locations. This was done by placing the stimulus at the other end of the apparatus over one of two possible food well locations and gently lifting the door, which started the trial. When presented with the rewarded object, food was located in the well underneath the object. When presented with the nonrewarded object, no food was located in the well beneath the object. Therefore, the rat should learn only to displace the object on rewarded trials. The latency from the opening of the door until the rat displaced the object was the dependent measure. If the rat did not displace the object after $10 \mathrm{sec}$, the trial was ended. Ten rewarded and 10 nonrewarded trials were given daily until the rats reached criterion level performance, defined as 3 consecutive days with a minimum of a $5 \mathrm{sec}$ difference in the latency scores between rewarded and nonrewarded trials.

Spatial location discrimination. This experiment required rats to discriminate between two spatial locations. One location was always paired with food, whereas the other spatial location was never paired with food, regardless of the object that occupied the spatial location. In this task, spatial location is important, and object information is irrelevant. On each trial, one of two different toy objects 
was used to define the spatial location. Each toy object was counterbalanced between both spatial locations. Other methods were identical to the object discrimination task, described above.

Object/spatial location paired-associate discrimination. This experiment required the rat to remember both object identity and spatial location information. The rat had to remember which object/spatial location pairs were associated with reward. In this task, both object and spatial location information are important. Two different objects and two spatial locations served as stimuli. One object was associated with food reward in one spatial location but not the other. The other object had the opposite pairing. Therefore, this task can be considered a paired-associate (PA) discrimination task for object and spatial location information. Other methods were identical to the object discrimination task described above.

\section{Lesion Analysis}

At the end of behavioral testing, all of the rats were deeply anesthetized with sodium pentobarbital and then transcardially perfused with saline, then with $4 \%$ phosphate-buffered paraformaldehyde. The brains were then removed, stored in a $30 \%$ glucose paraformaldehyde solution, and then cut in $30 \mu \mathrm{m}$ sections. Every fourth section was mounted onto glass slides and stained with cresyl violet. A projection microscope (Bausch \& Lomb) was used to examine the extent of the lesion.

\section{RESULTS}

\section{Histology}

Figure 1 presents a diagram of a representative parietal cortex control lesion across three anterior-posterior levels, based on the Swanson (1992) atlas. Although damage is not extensive, it is present. Figures 2 and 3 present diagrams of representative small and large PPC lesions, respectively, across three anterior-posterior levels, based on the Swanson (1992) atlas. Cortical damage observed in rats in the small PPC group was generally consistent across animals. Cortical damage in the large PPC lesions group was slightly more variable. Damaged areas always included the primary somatosensory area and the posterior parietal association area. In addition, some rats incurred damage to dorsal, primary, and ventral auditory areas.

\section{Behavior}

Figure 4 (A, B, and C) presents the mean number of trials $( \pm S E M)$ to reach criterion for each discrimination and clearly shows that rats with large and small lesions of the PPC are not impaired, relative to control rats in the object discrimination task or the spatial location discrimination task, but are impaired in the object plus spatial location discrimination task. A one-way analysis of variance (ANOVA) for the object discrimination task revealed no significant group differences $[F(2,14)=0.12$, $p>.05]$. A one-way ANOVA for the spatial location discrimination task also revealed no significant group differences $[F(2,14)=1.5, p>.05]$. A one-way ANOVA for the object and spatial location PA task revealed a significant group difference $[F(2,14)=46.5, p<.0001]$. A subsequent Newman-Keuls post hoc analysis on the numbers of trials to reach criterion on the object plus spatial location discrimination task revealed that both the small lesion group and the large lesion group were sig-
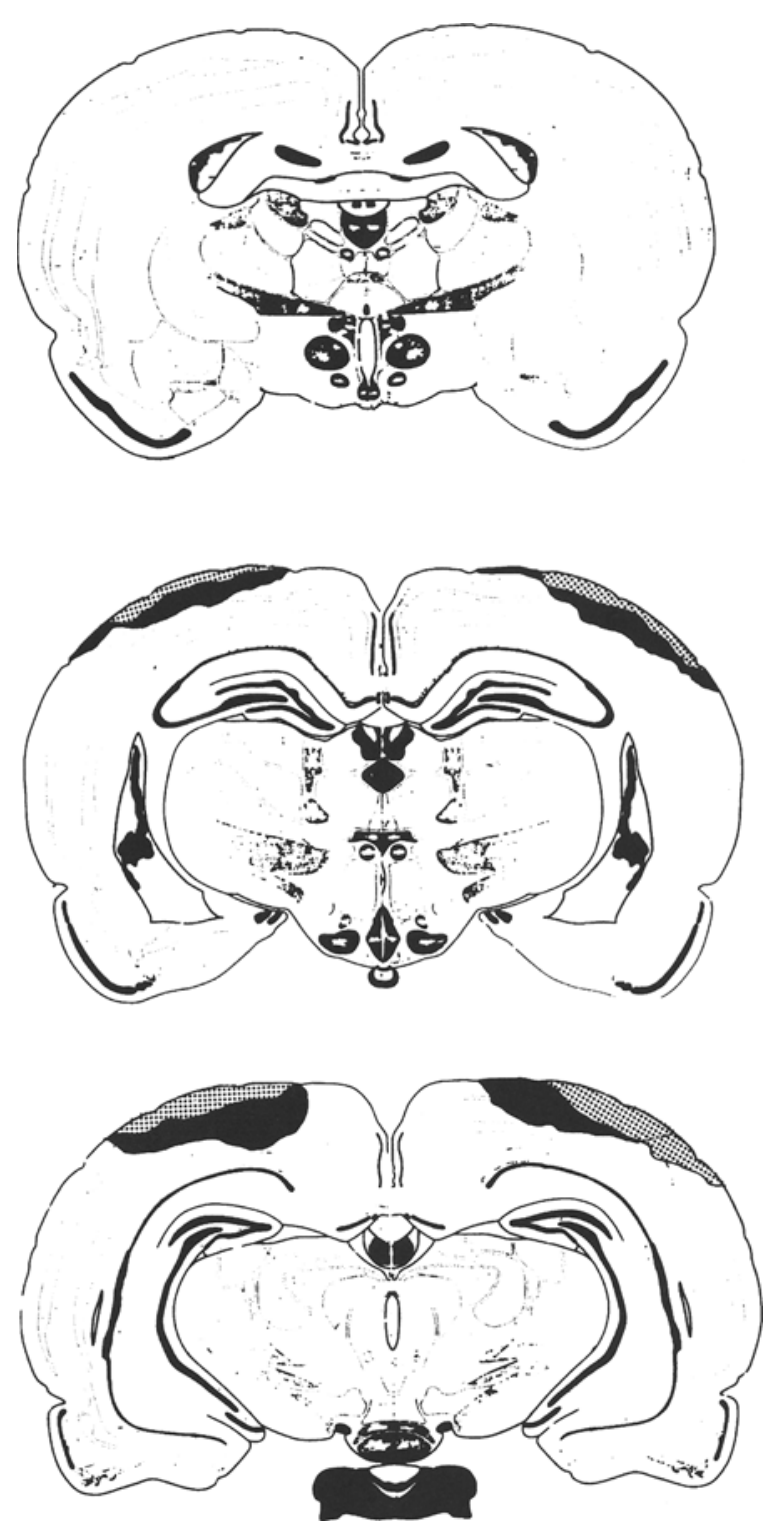

Figure 1. A schematic representation of the smallest (stippled) and largest (black) control lesion across three anterior-posterior levels, $-\mathbf{1 . 7 8},-3.90$, and $-\mathbf{5 . 0 0} \mathrm{mm}$ post-bregma, respectively.

nificantly different from the control group $(p<.05)$ and not significantly different from each other.

\section{DISCUSSION}

The results indicated that neither small nor large lesions of the rodent parietal cortex impaired the acquisition of either object or spatial location discrimination learning. However, both small and large lesions of the PPC impaired the acquisition of an object/spatial location PA task, a task that assessed the rats' memory for both object and spatial location information. The absence of a postlesion impairment in either the object only or the spatial 

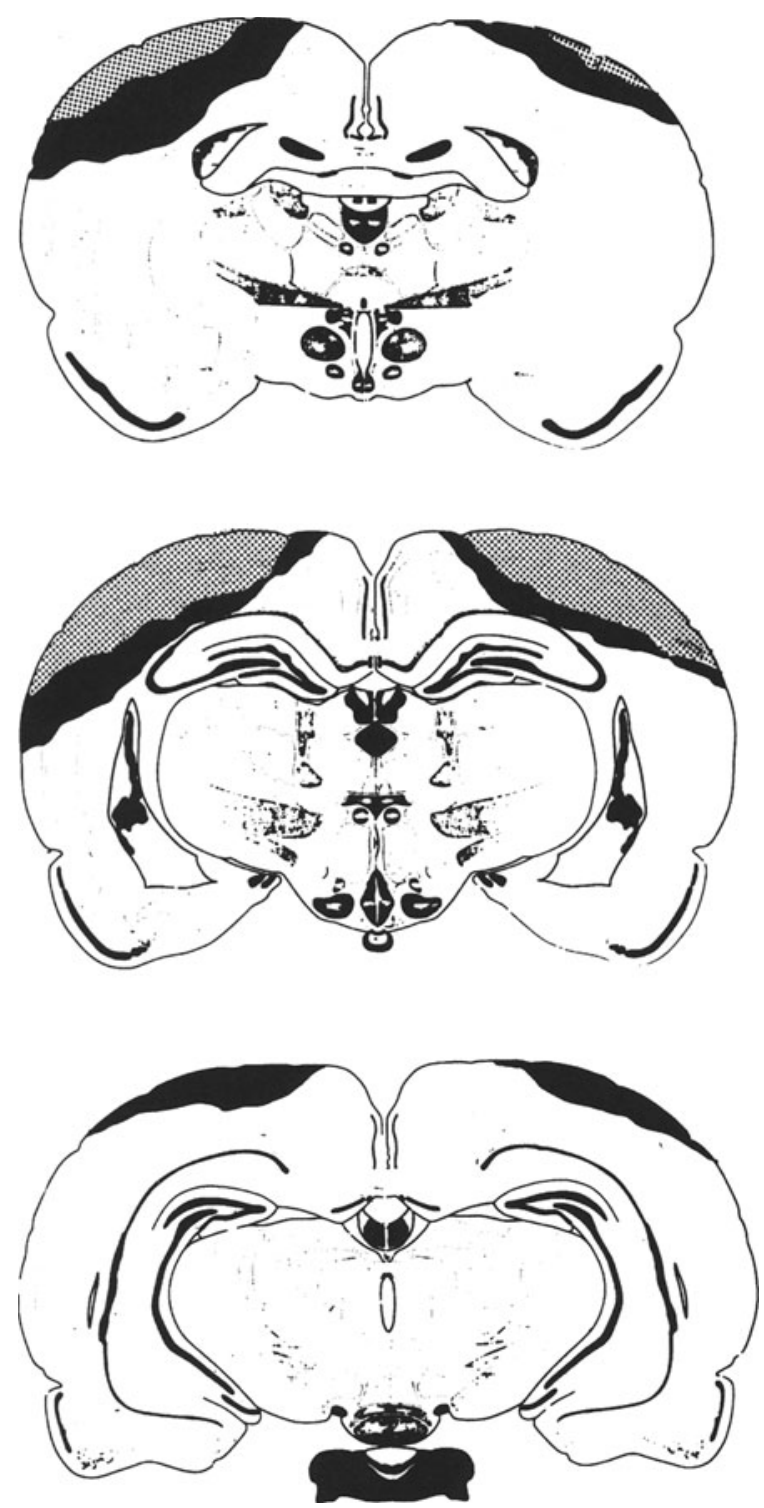

Figure 2. A schematic representation of the smallest (stippled) and largest (black) small posterior parietal cortex lesion across three anterior-posterior levels, $-1.78,-3.90$, and $-5.00 \mathrm{~mm}$ post-bregma, respectively.

location only discrimination tasks suggests that the underlying cause of the deficit observed in the object/spatial location PA task was not due to an inability to discriminate the individual elements but rather to an inability to keep a reference memory representation for the combination of the two elements.

It is possible that, if tested for a sufficient number of trials, the rats in the PPC lesion group could eventually learn the last task. The cutoff of 580 trials was chosen because it was twice the number of trials needed for the control group to reach criterion level performance. A subset of rats was tested up to 700 trials, but even then they did not reach criterion. This does not prove that PPC-lesioned rats could never reach criterion but supports the original claim that PPC rats are impaired, relative to controls.

Intact performance in the individual discriminations suggests that the deficit observed in the object/spatial location PA task was not due to lesion-induced changes in motivation, sensation, perception, or motor movement abilities. These data support the hypothesis that the rodent PPC is not involved in memory for a single landmark or a single spatial location, but rather in mediating processes associated with assigning a specific landmark to a specific spatial location. However, learning PA discriminations is arguably more complex than learning simple discriminations; operational support for this idea
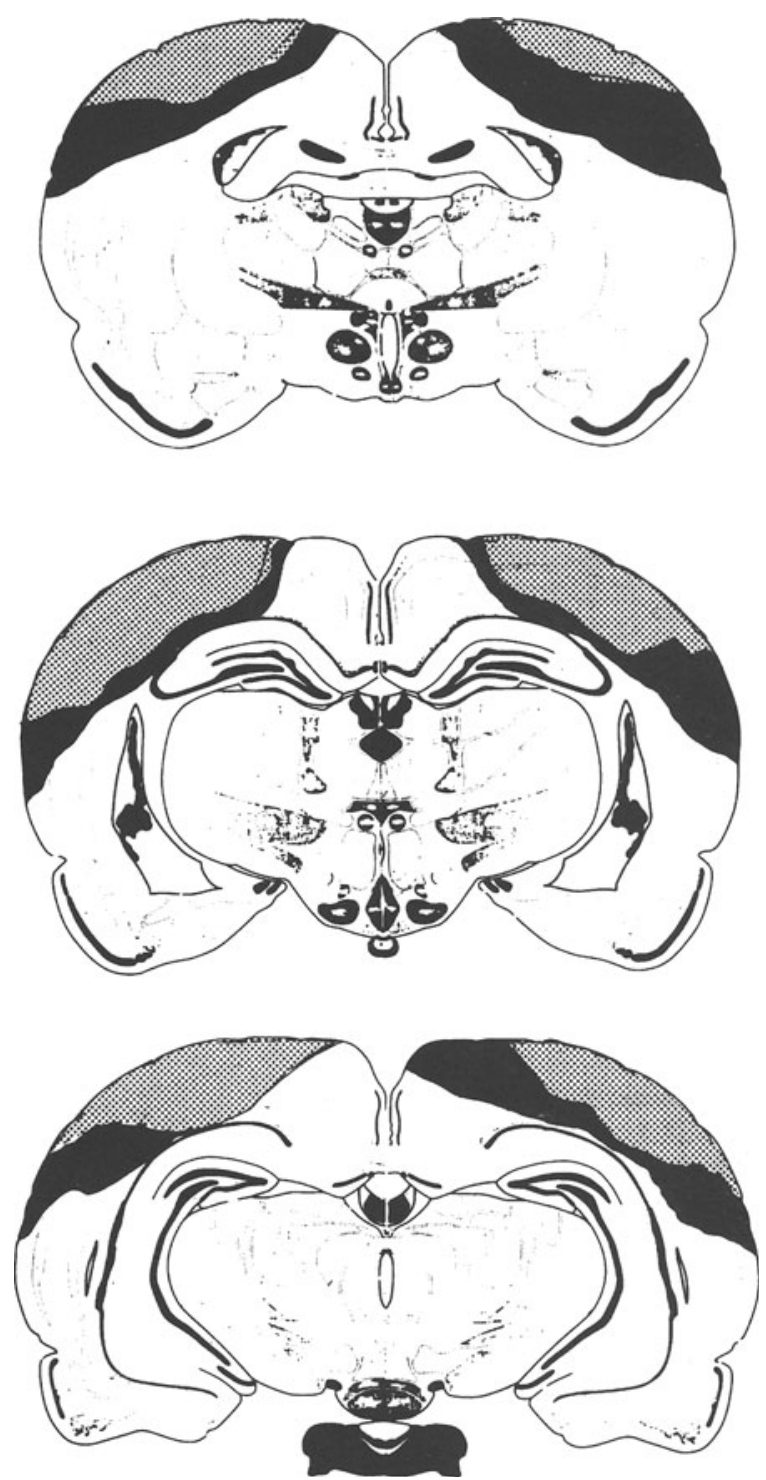

Figure 3. A schematic representation of the smallest (stippled) and largest (black) large posterior parietal cortex lesion across three anterior-posterior levels, $-\mathbf{1 . 7 8},-\mathbf{3 . 9 0}$, and $-\mathbf{5 . 0 0} \mathrm{mm}$ post-bregma, respectively. 
A

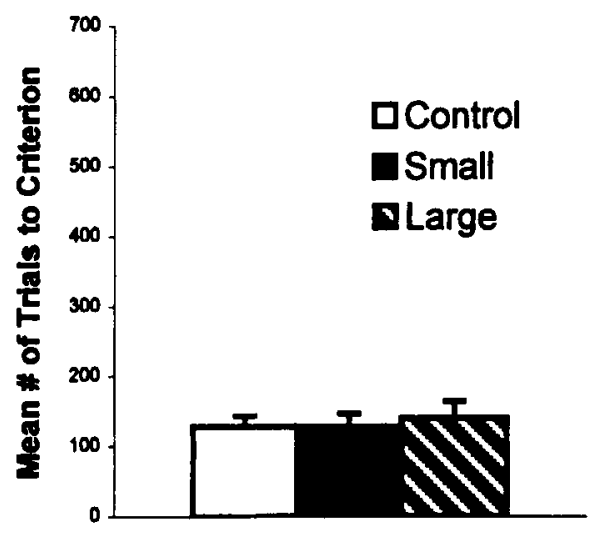

B

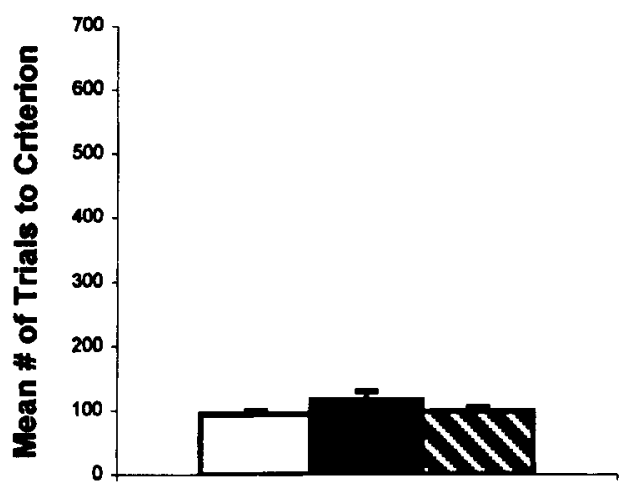

C

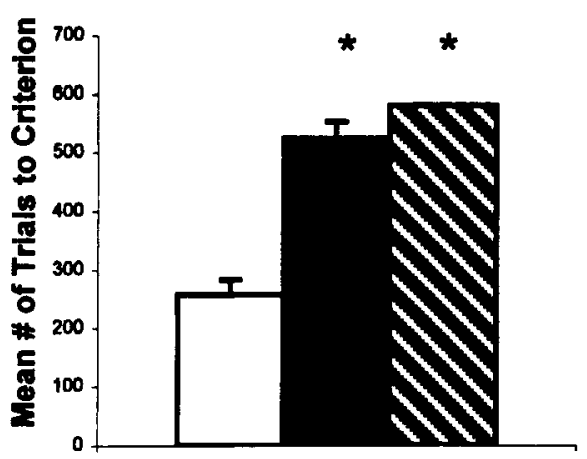

Figure 4. Mean number of trials to reach criterion $( \pm S E M)$ for the control, small posterior parietal, and large posterior parietal lesioned groups on object discrimination (A), spatial location discrimination (B), and object and spatial location discrimination (C).

is provided in the present experiment. The number of trials required by controls to reach criterion in the PA discrimination is a little more than double that required for the simple discriminations. This presents the possibility that deficits observed after PPC lesions are related to the difficulty of the task, independently of the features involved. Future work is needed to clarify this issue. However, data showing that lesions of the PPC do not impair retention of an object/object PA task (Long, Dial, \& Kesner, unpublished observations) suggest that the deficit observed in the present task is not due to an inability to process PA tasks in general or object/object PA specifically, but rather to an inability to hold a reference memory representation for the specific combination of object and spatial location information. This suggests that the PPC is involved in memory when associations among two (or more) stimuli are required and that at least one of the elements is spatial in nature. This is exactly one of the requirements thought necessary for the construction of a spatial cognitive map. Thus, impaired performance in the water maze after PPC lesions could be a result of the inability of the rat to maintain a memory for the combination of object (landmark) and spatial location information.

It is informative to note that PPC lesions in rats do not completely eliminate spatial learning. Either at the end of extensive spatial training (Kesner, Farnsworth, \& Kametani, 1991; Kolb \& Walkey, 1987) or repeated preexposure to the environment (McDaniel, Compton, \& Smith, 1994), rats with PPC lesions perform in a way that is comparable with that of controls. This suggests that, although the PPC is an important cortical region for spatial navigation, after extensive training, other, nonparietal brain regions are able to compensate. Again, it is possible that, with more training, the PPC-lesioned rats in the present experiment could have eventually learned the task.

These data are consistent with results obtained in humans with parietal cortex damage. For example, FriedmanHill et al. (1995) report a patient with symmetrical bilateral parieto-occipital damage who can discriminate shapes and letters but cannot correctly bind the color and size of two or more shapes. The subject experiences illusory conjunctions in which the memory for the color/ shape combination is incorrect. This could be analogous to the present experiment, in which the rats' memory for the object/spatial location combination is impaired. Although it should be noted that the subject in the FriedmanHill et al. study was impaired in processing spatial location information, the rats in the present experiment were not impaired in a simple spatial location discrimination task.

Furthermore, it has been suggested that, in humans, the left parietal cortex is involved in both object-based and space-based components of visual attention, whereas the right parietal cortex appears to be involved in only the spaced-based component of visual attention (Egly, Driver, \& Rafal, 1994). Finally, in humans, the activity of PPC (as measured by positron emission tomography) is not increased during a color or motion search task but is increased when subjects have to attend to the conjunction of color and motion (Corbetta, Shulman, Miezin, \& Petersen, 1995). In summary, there is behavioral and physiological evidence to support the result of the present experiment-mainly, that the PPC is involved in the memory for the conjunction of object and place information. 
The results also indicate that small lesions of the PPC, in the area designated as the rodent PPC by Reep et al. (1994), proved sufficient to impair performance in the object/spatial location PA task and, thus, are consistent with the Reep et al. description of the critical rodent PPC area. Moreover, the extra-PPC damage that occurred with the large lesion did not exacerbate the impairment seen with the small lesion. This suggests, but does not prove, that the nonparietal cortex region damaged in the large lesion group is not necessary to solve an object/spatial location PA task. However, preliminary work, damaging only the cortical region that was included in the large PPC lesion surgeries in the present experiment but not in the small PPC lesion surgeries, did not impair acquisition. In addition, other preliminary data suggest that lesions to the infralimbic and prelimbic regions also do not impair performance in the present task. These data provide support that specific regions of the central nervous system are involved in PA learning for object and place information, specifically the PPC. Future studies are needed to further explore this issue.

\section{REFERENCES}

ANDERSEN, R. A. (1987). Inferior parietal lobule function in spatial perception and visuomotor integration. In V. B. Mountcastle, F. Plum, \& S. R. Geiger (Eds.), Handbook of physiology: The nervous system $V$ (pp. 483-518). Baltimore: Williams \& Wilkins.

Chen, L. L,. Lin, L-H., Barnes, C. A., \& McNaughton, B. L. (1994). Head-direction cells in the rat posterior cortex: II. Contributions of visual and ideothetic information to the directional firing. Experimental Brain Research, 101, 24-34.

Chen, L. L., Lin, L-H., Green, E. J., Barnes, C. A., \& McNaughton, B. L. (1994). Head-direction cells in the rat posterior cortex: I. Anatomical distribution and behavioral modulation. Experimental Brain Research, 101, 8-23.

CRITCHLEY, M. (1953). The parietal lobes. London: Arnold.

Corbetta, M., Shulman, G. L, Miezin, F. M., \& Petersen, S. E. (1995). Superior parietal cortex activation during spatial attention shifts and visual feature conjunction. Science, 270, 802-805.

DeRENZI, E. (1982). Disorders of space exploration and cognition. New York: Wiley.

DiMATTIA, B. V., \& KESNER, R. P. (1988a). Role of the posterior parietal association cortex in the processing of spatial event information. Behavioral Neuroscience, 102, 397-403.

DiMATTIA, B. V., \& KeSNER, R. P. (1988b). Spatial cognitive maps: Differential role of parietal cortex and hippocampal formation. Behavioral Neuroscience, 102, 471-480.
Egly, R., DRIVER, J., \& Rafal, R. D. (1994). Shifting visual attention between objects and locations: Evidence from normal and parietal lesion subjects. Journal of Experimental Psychology: General, 123, 161-177.

Friedman-Hill, S. R., Robertson, L. C., \& Treisman, A. (1995). Parietal contributions to visual feature binding: Evidence from a patient with bilateral lesions. Science, 269, 853-855.

HYVÄRINEN, J. (1982). The parietal cortex of monkey and man. Berlin: Springer-Verlag.

Kesner, R. P., FARnsworth, G., \& Kametani, H. (1991). Role of parietal cortex and hippocampus in representing spatial information. Cerebral Cortex, 1, 367-373.

KING, V. R., \& CoRwIN, J. V. (1992). Spatial deficits and hemispheric asymmetries in the rat following unilateral and bilateral lesions of the posterior parietal or medial agranular cortex. Behavioural Brain Research, 50, 53-68.

Kolb, B., Buhrmann, K., McDonald, R., \& Sutherland, R. J. (1994). Dissociation of the medial prefrontal, posterior parietal, and posterior temporal cortex for spatial navigation and recognition memory in the rat. Cerebral Cortex, 6, 664-680.

KOLB, B., \& WALKEY, J. (1987). Behavioural and anatomical studies of the posterior parietal cortex in the rat. Behavioural Brain Research, 23, 127-145.

LONG, J. M., \& KESNER, R. P. (1996). The effects of dorsal vs. ventral hippocampal, total hippocampal, and parietal cortex lesions on memory for allocentric distance in rats. Behavioral Neuroscience, 110, 922-932.

LONG, J. M., \& KESNER, R. P. (in press). The effects of hippocampal and parietal cortex lesions on memory for egocentric distance and spatial location information in rats. Behavioral Neuroscience.

LYNCH, J. C. (1980). The functional organization of posterior parietal association cortex. Behavioral \& Brain Sciences, 3, 485-534.

McDaniel, W. F., Compton, D. M., \& SMith, S. R. (1994). Spatial learning following posterior parietal or hippocampal lesions. Cognitive Neuroscience \& Neuropathology, 5, 1713-1717.

McNaughton, B. L., Mizumori, S. J. Y., Barnes, C. A., Leonard, B. J., Marquis, M., \& GreeN, E. J. (1994). Cortical representation of motion during unrestrained spatial navigation in the rat. Cerebral Cortex, 4, 27-39.

O'KeEFE, J., \& NADEL, L. (1978). The hippocampus as a cognitive map. Oxford: Oxford University Press, Clarendon Press.

ReEP, R. L., Chandler, H. C., King, V, \& CoRwin, J. V. (1994). Rat posterior parietal cortex: Topography of corticocortical and thalamic connections. Experimental Brain Research, 100, 67-84.

SWANSON, L. W. (1992). Brain maps: Structure of the rat brain. Amsterdam: Elsevier.

Tolman, E. C. (1948). Cognitive maps in rats and men. Psychological Review, 55, 189-308.

(Manuscript received November 13, 1997; revision accepted for publication January 23, 1998.) 